\title{
Correlation Pattern Recognition in Nonoverlapping Scene Using a Noisy Reference
}

\author{
Pablo M. Aguilar-González and Vitaly Kober \\ Department of Computer Science, Centro de Investigación Científica y de Educación \\ Superior de Ensenada, \\ Km. 107 Carretera Tijuana-Ensenada, Ensenada 22860, B.C., México \\ \{paguilar, vkober\}@cicese.mx \\ http://www.cicese.mx/
}

\begin{abstract}
Correlation filters for recognition of a target in nonoverlapping background noise are proposed. The object to be recognized is given implicitly; that is, it is placed in a noisy reference image at unknown coordinates. For the filters design two performance criteria are used: signalto-noise ratio and peak-to-output energy. Computer simulations results obtained with the proposed filters are discussed and compared with those of classical correlation filters in terms of discrimination capability.
\end{abstract}

Keywords: correlation filters, pattern recognition.

\section{Introduction}

Since the pioneering work by VanderLugt [1, correlation filters have been extensively studied for the purpose of pattern recognition [2-15]. Within the context of pattern recognition, detection and location estimation are two very important tasks. When a correlation filter is used, such tasks may be solved in two steps; that is, first, the detection is carried out by searching the highest correlation peaks at the filter output, and then the coordinates of the peaks are taken as position estimations of targets in the scene image [2].

Different criteria have been proposed to evaluate the performance of correlation filters 3] such as signal-to-noise ratio (SNR), peak sharpness, light efficiency, discrimination capability, etc. Filters are designed by maximizing one of these criteria. Many filters have been proposed when an input scene contains a target distorted by additive noise. The matched filter (MF) [1] is derived by maximizing the SNR. The phase-only filter [4 maximizes light efficiency. The optimal filter (OF) 5] minimizes the probability of anomalous errors. Several filters have been derived for the nonoverlapping scene model 678910. The generalized matched filter [7] was derived by maximizing the ratio of the square of the expected value of the correlation peak to the average output variance. The generalized optimum filter 7] maximizes the peak-to-output energy ratio (POE). Recently [1], several correlation filters were proposed for the scene model that takes into account linear degradations of the both scene and target.

E. Bayro-Corrochano and J.-O. Eklundh (Eds.): CIARP 2009, LNCS 5856, pp. $555-562,2009$.
(C) Springer-Verlag Berlin Heidelberg 2009 
All of these filters, however, are derived under the assumption that a target is explicitly known. However, in real-life situations the target is often given by a reference image, which contains the reference object at unknown coordinates, as well as a noisy background. In a recent paper [12] a signal model was proposed that takes into account additive noise in the reference image to design filters for detecting a target in overlapping noise. The considered signal model is close to practical situations, in which observed and reference images are inevitably corrupted by noise owing to the image formation process. In this paper, extend that work to account for the presence of a nonoverlapping background in the input scene. We propose two correlation filters optimized with respect to the SNR and POE. The performance of the filters is compared to that of classical correlation filters.

\section{Analysis}

We use the additive signal model for the reference image and the nonoverlapping signal model for the input scene. For simplicity, 1-dimensional notation is used. Formally, the scene and reference image are given, respectively, by

$$
\begin{aligned}
s_{o}(x) & =t\left(x-x_{s}\right)+b(x) \bar{w}\left(x-x_{s}\right)+n_{s}(x), \\
r(x) & =t\left(x-x_{r}\right)+n_{r}(x),
\end{aligned}
$$

where $t(x)$ is the target, $s_{o}(x)$ is the observed scene with the target location $x_{s}, r(x)$ is the reference image with the target located at the coordinate $x_{r}$, and $n_{s}(x)$ and $n_{r}(x)$ are noise signals in the input scene and the reference image, respectively. $b(x)$ is the nonoverlapping background, treated as the realization of a stationary random process with the mean $\mu_{s}$ and the power spectral density $B_{0}(\omega)$, and it is multiplied by $\bar{w}(x)$ the inverse support function of the target. Both $n_{s}(x)$ and $n_{r}(x)$ are assumed to be stationary random processes. It is also assumed that the random processes and the random target locations $x_{s}$ and $x_{r}$ are statistically independent of each other. We will design filters to be applied to the centered scene, that is $s(x)=s_{o}(x)-\mu_{s} . S(\omega)$ and $T(\omega)$ are the Fourier transforms of $s(x)$ and $t(x)$, respectively, and $N_{s}(\omega)$ and $N_{r}(\omega)$ are the power spectral densities of $n_{s}(x)$ and $n_{r}(x)$, respectively.

Since the target signal is not available, we look for a correlation filter of the following form:

$$
H(\omega)=A(\omega) R^{*}(\omega),
$$

where $A(\omega)$ is a deterministic function, $R(\omega)$ is the Fourier transform of the realization of the reference image given in (2), and * denotes complex conjugate. Actually, it is interesting to note that the filter is given by a bank of the transfer functions determined by a realization of the random process $n_{r}$.

Because the location of the target in the reference image is $x_{r}$ and not the origin, the correlation output peak is expected at the coordinate $x_{s}-x_{r}$. Note however that as long as the target is reasonably centered in the reference image, the location estimation of the target in the input scene will be in the vicinity of 
the true location. Even if the exact location of the target can't be determined, knowing the relative position is useful for applications like tracking [16], where what is important is the tracked object's trajectory.

The modified generalized optimum filter $\left(\mathrm{GOF}_{\mathrm{AN}}\right)$ is derived by maximizing the POE criterion:

$$
\mathrm{POE}=\frac{\left|\mathrm{E}\left\{y\left(x_{s}-x_{r}\right)\right\}\right|^{2}}{\mathrm{E}\left\{\overline{|y(x)|^{2}}\right\}},
$$

where $\mathrm{E}\{\cdot\}$ denotes the expected value and and the over-bar denotes spatial averaging, i.e. $\overline{y(x)}=(1 / L) \int y(x) d x, L$ is the spatial extent of the signal $y(x)$. The expected filter output at the location of the correlation peak can be calculated as

$$
\mathrm{E}\left\{y\left(x_{s}-x_{r}\right)\right\}=\frac{1}{2 \pi} \int A(\omega) \mathrm{E}\left\{\left(R(\omega) e^{i \omega x_{r}}\right)^{*} S(\omega) e^{i \omega x_{s}}\right\} d \omega .
$$

We can calculate $\mathrm{E}\left\{\overline{|y(x)|^{2}}\right\}$ as

$$
\mathrm{E}\left\{\overline{|y(x)|^{2}}\right\}=\frac{1}{2 \pi L} \int|A(\omega)|^{2} \mathrm{E}\left\{\left|R^{*}(\omega) S(\omega)\right|^{2}\right\} d x .
$$

Substituting (5) and (6) into (4) we get

$$
\mathrm{POE}=\frac{L\left|\int A(\omega) \mathrm{E}\left\{\left(R(\omega) e^{i \omega x_{r}}\right)^{*} S(\omega) e^{i \omega x_{s}}\right\} d \omega\right|^{2}}{2 \pi \int|A(\omega)|^{2} \mathrm{E}\left\{\left|R^{*}(\omega) S(\omega)\right|^{2}\right\} d x},
$$

and applying the Cauchy-Schwartz inequality, the expression for the $\mathrm{GOF}_{\mathrm{AN}}$ is given by

$\operatorname{GOF}_{\mathrm{AN}}(\omega)=\frac{\left(|T(\omega)|^{2}-\mu_{s} T(\omega) W^{*}(\omega)\right) R^{*}(\omega)}{\left(|T(\omega)|^{2}+N_{r}(\omega)\right)\left(\left|T_{s}(\omega)\right|^{2}+\frac{1}{2 \pi} B_{0}(\omega) *|\bar{W}(\omega)|^{2}+N_{s}(\omega)\right)}$,

where $T_{s}(\omega)$ is the Fourier transform of the expected value of the centered input scene, namely $t_{s}(x)=t(x)-\mu_{s} w(x) . w(x)$ is the support function for the target, that is $w(x)=1-\bar{w}(x)$.

The modified matched filter $\left(\mathrm{GMF}_{\mathrm{AN}}\right)$ can be derived by maximizing the SNR criterion:

$$
\mathrm{SNR}=\frac{\left|\mathrm{E}\left\{y\left(x_{s}-x_{r}\right)\right\}\right|^{2}}{\overline{\operatorname{Var}\{y(x)\}}},
$$

where $\operatorname{Var}\{\cdot\}$ denotes variance. The variance of the output is given by

$$
\overline{\operatorname{Var}\{y(x)\}}=\frac{1}{2 \pi L} \int|A(\omega)|^{2} \operatorname{Var}\left\{R^{*}(\omega) S(\omega)\right\} d \omega .
$$


Substituting (5) and (10) into (9) we obtain the following expression for the SNR:

$$
\mathrm{S} \tilde{\mathrm{NR}}=\frac{L\left|\int A(\omega) \mathrm{E}\left\{\left(R(\omega) e^{i \omega x_{r}}\right)^{*} S(\omega) e^{i \omega x_{s}}\right\} d \omega\right|^{2}}{2 \pi \int|A(\omega)|^{2} \operatorname{Var}\left\{R^{*}(\omega) S(\omega)\right\} d \omega} .
$$

Applying the Cauchy-Schwartz inequality we obtain the expression for the GMF $A N$

$$
\operatorname{GMF}_{\text {AN }}(\omega)=\frac{\left(|T(\omega)|^{2}-\mu_{s} T(\omega) W^{*}(\omega)\right) R^{*}(\omega)}{\left(|T(\omega)|^{2}+N_{r}(\omega)\right)\left(\left|T_{s}(\omega)\right|^{2}+\frac{1}{2 \pi} B_{0}(\omega) *|\bar{W}(\omega)|^{2}+N_{s}(\omega)\right)-\left|T(\omega) T_{s}(\omega)\right|^{2}} .
$$

Note that if the reference image does not contain noise, the $\mathrm{GMF}_{\mathrm{AN}}$ and the $\mathrm{GOF}_{\mathrm{AN}}$ are equal to the classical GMF and GOF, respectively.

It can be seen that the obtained filters require knowledge of the target Fourier transform, and the support function. This contradicts the assumption that information about the target is unknown. However, estimations may be designed from the available information. We can apply the smoothing Wiener filter [17] to attenuate the effects of noise in the reference image. After that, we can apply a threshold to the resulting image and obtain an approximate support function.

$$
\begin{aligned}
& \tilde{r}(x)=r(x) * h_{\text {wiener }}(x), \\
& \tilde{w}(x)= \begin{cases}1 & \tilde{r}(x) \geq \tau\left(\mu_{t}, \sigma_{t}, \sigma_{n_{r}}\right) \\
0 & \text { otherwise }\end{cases}
\end{aligned}
$$

The optimum threshold depends on the statistics of the input noise and the target. When the target mean and standard deviation are known, these values can be used to improve the threshold selection. If such values are unknown, the optimum threshold can be determined in terms of the input noise statistics. If the support function estimation is not reliable owing to the presence of high levels of noise in the reference image, we can approximate the optimum filter transfer function by disregarding terms that require the support function. Figure 1] shows the regions of the parameter space in which each estimation is best. When SNR is high, we can correctly estimate the support function and use that with the original reference image. When SNR is not high enough, it is better to use the Wiener filtered reference image. And in some cases, when the input SNR is low and the target occupies a large percent of the reference image, it is better to not consider the estimated support function because it introduces errors inside the area occupied by the target. In these cases the noise present outside of the target is small and affects performance less than an incorrect estimation of the support function. Target image estimations based on the three regions are given as follows:

$$
\tilde{t}(x)= \begin{cases}\tilde{r}(x), & \text { if input SNR is low and target is large } \\ \tilde{r}(x) \tilde{w}(x), & \text { if input SNR is low and target is small } . \\ r(x) \tilde{w}(x), & \text { if input SNR is high }\end{cases}
$$




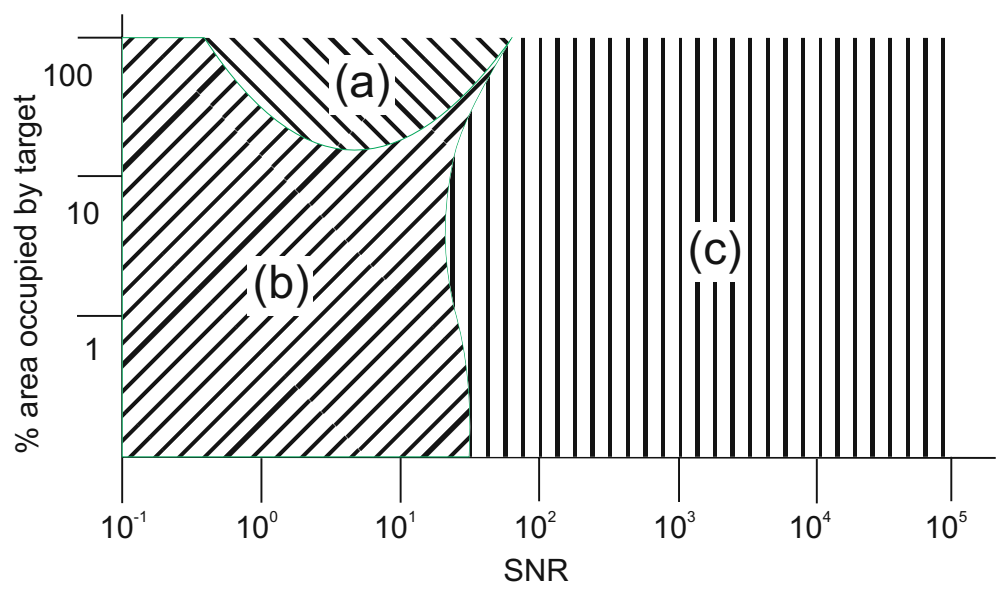

Fig. 1. Regions of the SNR-Area parameter space where each estimation is best: (a) better not to estimate the support function, (b) better to estimate target using filtered reference image, and (c) better to estimate target using estimated support function and the original reference image

\section{Computer Simulations}

In this section we present computer simulation results. The performance of the proposed filters are compared with that of the classical filters in terms of the discrimination capability (DC). The DC is formally defined [5] as the ability of a filter to distinguish a target from other objects in the scene. The DC can be expressed as follows:

$$
D C=1-\frac{\left|C^{B}(0)\right|^{2}}{\left|C^{T}(0)\right|^{2}},
$$

where $\left|C^{B}(0)\right|$ is the maximum value in the correlation plane over the background area, and $\left|C^{T}(0)\right|$ is the maximum value in the correlation plane over the area the target occupies in the input scene. The background area and the target area are complementary. Values of the DC close to unity, indicate a good capacity to discriminate the target against unwanted objects. Negative values of the DC indicate a failure to detect the target. We show simulation results when using generalized optimum filters only. Generalized matched filters do not control the output mean value which may result in a correlation peak being buried in output noise that has a high mean [7] and thus perform poorly in terms of DC.

The size of all images used in the experiments is $256 \times 256$ pixels. All filters are implemented using the Discrete Fourier Transform. The intensity values are in the range [0-255]. We use the butterfly shown in Fig. 2(a) as a target. There are two background types, shown in Fig. 22 deterministic and stochastic backgrounds. The stochastic background is a realization of a colored random process 
with the mean and standard deviation of 115 and 40, respectively, and with the horizontal and vertical correlation coefficients of 0.95 . To guarantee statistically correct results, 30 statistical trials of each experiment for either different positions of the target or realizations of random processes were performed. The sample reference images are corrupted by additive white Gaussian noise.

Three filters are used in the experiments: the classical GOF which is designed with all parameters known to establish an upper bound on performance; the proposed $\mathrm{GOF}_{\mathrm{AN}}$ filter when estimating the target as $r(x) \tilde{w}(x)$, shown as $\mathrm{GOF}_{\mathrm{AN}} 1$ in the simulation results; and the proposed filter when estimating the target as $\tilde{r}(x) \tilde{w}(x)$, shown as $\mathrm{GOF}_{\mathrm{AN}} 2$.

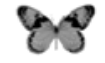

(a)

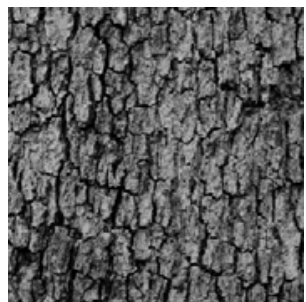

(b)

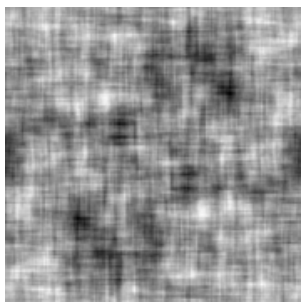

(c)

Fig. 2. (a) The target used in the experiments, (b) deterministic background, (c) example of stochastic background

\subsection{Scenario 1: Stochastic Background}

In order to determine the performance of the proposed filters, we performed experiments for different realizations of the background process while the location of the target within the scene was varied. The simulation results are shown in Fig. 3. It can be seen that the performance for the GOF remains constant. It is because this filter is designed with all parameters known and no noise presence. It can also be seen that the proposed filters are able to detect the target even in the presence of noise of a Std. Dev. of up to 20. When there are higher levels of noise in the reference image performance drops quickly. This is because we are unable to estimate the target support function and we also can not design the filter by ignoring the support function, because the target information is mostly destroyed by the presence of noise. It can be seen that performance behaves similarly regardless of the level of noise present in the input scene.

\subsection{Scenario 2: Deterministic Background}

We also test the performance of the proposed filters in when the background is a deterministic scene. The results are shown in Fig. 4. In this scenario the performance of the GOF also remains constant because it is not affected by the reference image noise. For the proposed filters, the mean value of the DC decreases as the noise in the reference image surpasses the same threshold as in 


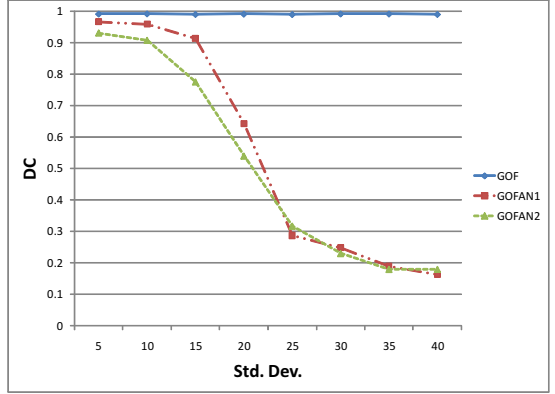

(a)

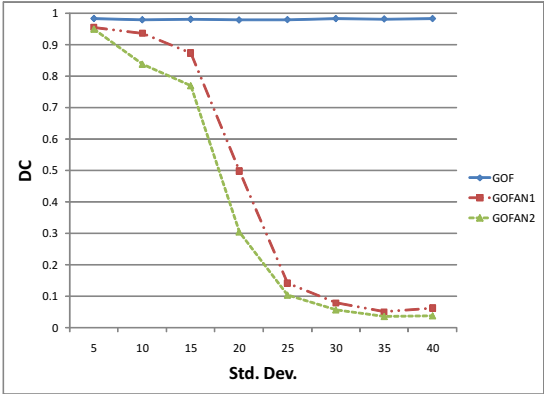

(b)

Fig. 3. Performance of correlation filters in terms of DC while varying the Std. Dev. of the reference image noise. The scene has a stochastic background and additive scene noise Std. Dev. of (a) 5 and (b) 15.

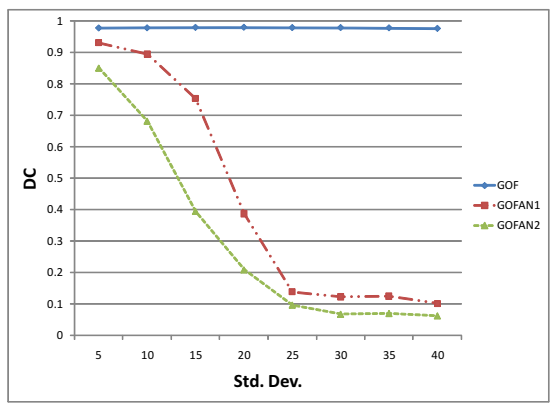

(a)

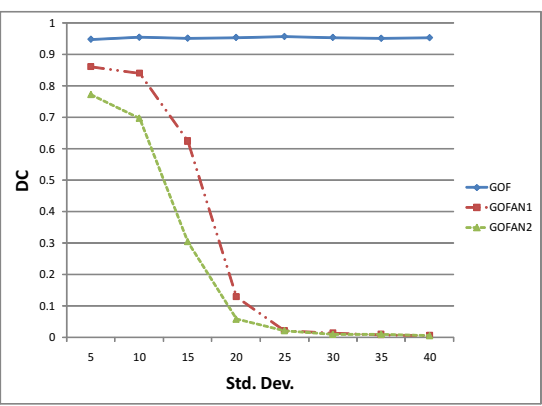

(b)

Fig. 4. Performance of correlation filters in terms of DC while varying the Std. Dev. of the reference image noise. The scene has a deterministic background and additive scene noise Std. Dev. of (a) 5 and (b) 15.

scenario 1. Detection performance decreases more as the additive noise in the input scene increases but the behavior remains the same.

\section{Conclusion}

In this paper new correlation filters for recognition of a target in nonoverlapping background noise were proposed. The filters are derived from a new reference model, which takes into account the presence of additive noise in the reference image. With the help of computer simulations, we showed that the proposed filters yield good results in the presence of moderate levels of noise. It was also shown that the proposed filters are robust to different realizations of the reference image noise. 


\section{References}

1. VanderLugt, A.B.: Signal Detection by Complex Filtering. IEEE Trans. Inf. Theory IT 10, 139-145 (1964)

2. Vijaya-Kumar, B.V.K., Mahalanobis, A., Juday, R.D.: Correlation Pattern Recognition. Cambridge University Press, Cambridge (2005)

3. Vijaya-Kumar, B.V.K., Hassebrook, L.: Performance Measures for Correlation Filters. Appl. Opt. 29, 2997-3006 (1990)

4. Horner, J.L., Gianino, P.D.: Phase-Only Matched Filtering. Appl. Opt. 23, 812-816 (1984)

5. Yaroslavsky, L.P.: The Theory of Optimal Methods for Localization of Objects in Pictures. In: Wolf, E. (ed.) Progress in Optics, vol. 33, pp. 145-201. Elsevier, Amsterdam (1993)

6. Javidi, B., Wang, L.: Optimum Filter for Detection of a Target in Nonoverlapping Scene Noise. Appl. Opt. 33, 4454-4458 (1994)

7. Javidi, B., Wang, J.: Design of Filters to Detect a Noisy Target in Nonoverlapping Background Noise. J. Opt. Soc. Am. A 11, 2604-2612 (1994)

8. Réfrégier, P., Javidi, B., Zhang, G.: Minimum Mean Square Error Filter for Pattern Recognition With Spatially Disjoint Signal and Scene Noise. Opt. 18, 1453-1455 (1993)

9. Javidi, B., Horner, J.L.: Real-Time Optical Information Processing. Academic Press, Inc., London (1994)

10. Kober, V., Campos, J.: Accuracy of Location Measurement of a Noisy Target in a Nonoverlapping Background. Journal OSA 13, 1653-1666 (1996)

11. Ramos, E.M., Kober, V.: Design of Correlation Filters for Recognition of Linearly Distorted Objects in Linearly Degraded Scenes. J. Opt. Soc. Am. A 24, 3403-3417 (2007)

12. Aguilar-González, P.M., Kober, V.: Correlation Filters for Pattern Recognition Using a Noisy Reference. In: Ruiz-Shulcloper, J., Kropatsch, W.G. (eds.) CIARP 2008. LNCS, vol. 5197, pp. 38-45. Springer, Heidelberg (2008)

13. Kober, V., Ovseyevich, A.: Phase-Only Filter with Improved Filter Efficiency and Correlation Discrimination. Pattern Recognition and Image Analysis 10, 514-519 (2000)

14. Díaz-Ramírez, V.H., Kober, V., Álvarez-Borrego, J.: Pattern Recognition With an Adaptive Joint Transform Correlator. Appl. Opt. 45, 5929-5941 (2006)

15. González-Fraga, J.A., Kober, V., Álvarez-Borrego, J.: Adaptive Synthetic Discriminant Function Filters for Pattern Recognition. Opt. Eng. 45, 1-10 (2006)

16. Yilmaz, A., Javed, O., Shah, M.: Object tracking: A survey. ACM Comput. Surv. 38(4), Article 13 (2006)

17. Pratt, W.K.: Digital Image Processing. John Wiley \& Sons, Chichester (2001) 